\title{
Compatibility Relations and the Finite-Element Formulation of Electromagnetic Field Problems
}

\author{
Gerrit Mur \\ Faculty of Electrical Engineering \\ Delft University of Technology \\ P.O. Box 5031, 2600GA Delft, The Netherlands
}

\begin{abstract}
When computing an electromagnetic field using the finite element method it is possible that, although Maxwell's equations are discretized accurately, highly inaccurate computational results are obtained. In those cases it can easily be shown that (some of) the electromagnetic compatibility relations (field properties that follow from Maxwell's equations) are not satisfied. The divergence condition on the fluxes, for instance, follows directly from the field equations but not necessarily from their discretized counterparts. This necessitates inclusion of the compatibility relations in the finite-element formulation of the field problem. A survey is given of all electromagnetic compatibility relations.
\end{abstract}

\section{INTRODUCTION}

Because of its flexibility, the finite-element method seems to be the most suitable method for computing electromagnetic fields in inhomogeneous media and/or complicated geometries. In the finite-element formulation of an electromagnetic field problem the field equations can only be satisfied approximately. As a consequence of this, field properties that follow from Maxwell's electromagnetic field equations, the electromagnetic compatibility relations [1], may not be reflected accurately in a numerical solution. In earlier papers $[2,3]$ we presented methods for computing the electric and/or the magnetic field directly, using a combination of linear edge and linear nodal expansion functions for obtaining optimum computational efficiency. In these papers, we mentioned the importance of including the divergence condition, which is one of the compatibility relations to be discussed, in the formulation of the problem. The equations applying to the divergence of the electric and magnetic flux densities follow directly from the electromagnetic field equations. They are satisfied whenever the field equations are satisfied exactly.

In the present paper the use of the divergence condi-

Manuscript received November 1,1993. tion will be generalized to the use of the compatibility relations for electromagnetic fields. It will also be shown that the use of divergence-free edge elements, which is advocated by some authors (see [4] and the references contained in it) for satisfying some of these compatibility relations, as well as the use of face elements, introduces the possibility of violating additional relations of the compatibility type. The importance of including the electromagnetic compatibility relations explicitly in the finite-element formulation of the problem is stressed. The analysis of the compatibility relations is carried out for methods for computing time-domain (transient) electromagnetic fields. The analysis of methods for timeharmonic electromagnetic as well as for static electric or magnetic field problems runs along similar lines and leads to similar conclusions.

\section{THE BASIC EQUATIONS}

As the point of departure for our analysis we use the time-domain electromagnetic field equations

$$
\begin{aligned}
& \partial_{t} \boldsymbol{D}+\boldsymbol{J}-\nabla \times \boldsymbol{H}=-\boldsymbol{J}^{\mathrm{imp}}, \\
& \partial_{t} B \quad+\nabla \times E=-K^{\text {imp }} \text {, }
\end{aligned}
$$

where $\boldsymbol{J}^{\mathrm{imp}}$ and $\boldsymbol{K}^{\mathrm{imp}}$ are imposed sources of electric and magnetic current that are known, throughout the domain of computation $\mathcal{D}$, as a function of the time coordinate $t$. The field equations are supplemented by the boundary conditions

$$
\begin{aligned}
\nu \times E & =\nu \times E^{\text {ext }} \text { on } \partial \mathcal{D}_{\mathrm{E}}, \\
\nu \times \boldsymbol{H} & =\boldsymbol{\nu} \times \boldsymbol{H}^{\text {ext }} \text { on } \partial \mathcal{D}_{\mathrm{H}},
\end{aligned}
$$

where $\nu$ is the unit vector along the normal to the outer boundary $\partial \mathcal{D}=\partial \mathcal{D}_{\mathrm{E}} \cup \partial \mathcal{D}_{\mathrm{H}}$ (with $\partial \mathcal{D}_{\mathrm{E}} \cap \partial \mathcal{D}_{\mathrm{H}}=\emptyset$ ) of the domain of computation $\mathcal{D}$, and where $\boldsymbol{E}^{\text {ext }}$ and $\boldsymbol{H}^{\text {ext }}$ are known, along the relevant parts of this outer boundary, as a function of $t$. Together with the constitutive equations and the initial conditions at $t=t_{0},(1)-(4)$ define an electromagnetic-field problem with a unique solution [5]. 
Note that the imposed source terms in (1) and (2) are not related to the known external fields in (3) and (4).

\section{ThE COMPATIBILITY RELATIONS}

Compatibility relations [1] are properties of a field that are direct consequences of the field equations and that must be satisfied to allow them to have a solution. For the electromagnetic field equations they are discussed below.

\section{A. Interior}

Applying the divergence operator $\nabla \cdot$ to (1) and (2) it follows that

$$
\begin{gathered}
\nabla \cdot\left(\partial_{t} D+J\right)=-\nabla \cdot J^{\mathrm{imp}}, \\
\partial_{t} \nabla \cdot \boldsymbol{B}=-\nabla \cdot \boldsymbol{K}^{\mathrm{imp}} .
\end{gathered}
$$

The electromagnetic compatibility (divergence) relations (5) and (6) apply to subdomains of the domain of computation in which the electromagnetic field vectors are continuously differentiable functions of the spatial coordinates.

Note that these relations are, contrary to what is stated by some authors [6], no additional (independent) equations, they are direct consequences of the field equations.

\section{B. Interfaces}

The field vectors are not differentiable with respect to the spatial coordinates at the interfaces between regions with different medium properties. In that case (5) and (6) are replaced by

$$
\begin{gathered}
\nu \cdot\left(\partial_{t} D+J\right)+\nu \cdot J^{\text {imp }} \text { continuous across interface, } \\
\boldsymbol{\nu} \cdot \partial_{t} B+\boldsymbol{\nu} \cdot \boldsymbol{K}^{\text {imp }} \text { continuous across interface, }
\end{gathered}
$$

where $\nu$ is the unit vector normal to the interface.

Note that (7) and (8) express the continuity condition applying to the normal components of the electric and the magnetic flux densities across an interface between different media.

\section{Outer boundary}

A third type of compatibility relation is found when studying the behavior of the field near the outer boundary of the domain of computation. Applying the operator $\nu$, where $\nu$ denotes the unit vector along the normal to the outer boundary, to (1) and (2) we obtain, using (3) and (4), the relations

$$
\begin{gathered}
\nu \cdot\left(\partial_{t} D+J\right)=\nu \cdot\left(\nabla \times H^{\text {ext }}-J^{\text {imp }}\right) \text { on } \partial \mathcal{D}_{\mathrm{H}} \\
\nu \cdot \partial_{t} B=-\nu \cdot\left(\nabla \times E^{\text {ext }}+K^{\text {imp }}\right) \text { on } \partial \mathcal{D}_{\mathrm{E}}
\end{gathered}
$$

These equations express the fact that prescribing the tangential components of the electric (magnetic) field strength at a given part of the outer boundary implies a related behavior of the normal components of the magnetic (electric) flux densities at that part of the boundary.

Note that these equations have the form of additional boundary conditions applying at the outer boundary of the domain of computation. They follow, however, directly from the fact that the field inside the domain of computation should satisfy Maxwell's equations.

\section{Compatibility relations and edge elements}

Some authors use divergence-free edge elements (e.g. "Whitney 1") for imposing the divergence conditions exactly. Apart from the fact that such an approach can only be used in the simple case where the compatibility relations (5) and (6) reduce to $\nabla \cdot D=0$ and $\nabla \cdot B=0$, respectively, edge elements have the disadvantage of causing the normal component of the field at the interface between two adjoining tetrahedra to be free to jump, even when it should be continuous. As a consequence of this, the continuity of the normal flux densities in between adjoining edge elements containing identical materials is guaranteed no longer, and the latter condition has to be added to our list of compatibility relations to be imposed upon the solution. Failing to do so may be the cause of undesired surface charge distributions in between edge elements. When adjoining finite elements contain identical materials, and assuming that the external sources of current are continuous between those finite elements, the following relations hold

$$
\begin{aligned}
& \boldsymbol{\nu} \cdot \boldsymbol{E} \text { continuous between edge elements, } \\
& \boldsymbol{\nu} \cdot \boldsymbol{H} \text { continuous between edge elements. }
\end{aligned}
$$

In the alternative cases, (7) and (8) still apply. Imposing these conditions results in an increase of the connectivity of the system matrices. Note that the need for imposing the continuity relations (11) and (12) is caused solely by the use of edge expansion functions and not by the electromagnetic field problem or the formulation used.

\section{E. Compatibility relations and face elements}

Some authors propose the use of face (also called facet) elements for modeling flux distributions. Face elements 
cause the normal fluxes between tetrahedra to be continuous, but they have the disadvantage of leaving tangential components to be free to jump, even when they should be continuous. When adjoining finite elements contain identical materials, and assuming that no surface sources of current are present at the interface between those finite elements, the following continuity relations should hold

$$
\begin{aligned}
& \boldsymbol{\nu} \times \boldsymbol{E} \text { continuous between face elements, } \\
& \boldsymbol{\nu} \times \boldsymbol{H} \text { continuous between face elements. }
\end{aligned}
$$

Imposing these conditions results in an increase of the connectivity of the system matrix (matrices). Note that the need for imposing the continuity relations (13) and (14) is caused solely by the use of face expansion functions and not by the electromagnetic field problem or the formulation used.

\section{F. In summary}

Equations (5)-(10) are a set of six electromagnetic compatibility relations that are direct consequences of Maxwell's equations. In exact methods for solving the electromagnetic field equations they are automatically accounted for. In numerical methods, for instance in the finite-element method, for solving the electromagnetic field equations they should be taken into account explicitly whenever the method used does not automatically account for them.

Equations (11)-(14) are additional compatibility relations the need for which is caused by the use of either edge or face elements in homogeneous domains. In those domains edge and face elements allow unphysical discontinuities in the solution and compatibility relations have to be added to the formulation of the field problem for restricting those discontinuities to acceptable values.

Note that (5)-(14) do not contain any extra information that is not contained in the field equations. However, failing to include them in the finite-element formulation of an electromagnetic-field problem, either exactly or numerically, may be the cause of highly inaccurate results. Errors of this type are often referred to as "spurious solutions" or "vector parasites".

\section{SOME APPLICATIONS}

To illustrate the importance of including the compatibility relations in the finite-element formulation of electromagnetic field problems we discuss some examples. Because of the limited amount of space available, we cannot give explicit examples of the application of each of the relations given. Our examples are all taken from the literature where particular (combinations of) compatibility relations are used.

\section{A. Interior compatibility}

The conditions of zero divergence, $\nabla \cdot \boldsymbol{D}=\mathbf{0}$ and $\nabla \cdot B=0$, are the best known examples of the compatibility relations, they are simple cases of (5) and (6). Examples of their importance in time-harmonic problems are discussed in [7]. The importance of including this condition in time-harmonic applications increases with decreasing angular frequency. Failing to include the interior compatibility condition in codes for transient problems nearly always causes instabilities.

\section{B. Interface compatibility}

The importance of satisfying the divergence condition applying to interfaces is stressed in $[8,9]$. The accuracy of satisfying the interface condition improves significantly when using them.

Singularities caused by interfaces that are locally not flat are often the cause of large errors in the normal flux continuity near those singularities. Especially in those cases, imposing the normal flux continuity is of great importance.

\section{Outer boundary compatibility}

The only example known to the author of the application of the compatibility relations for transient problems, which includes the one applying to the outer boundary, is found in [10]. For static problems, however, the static versions of (9) and (10) are the natural choices and well known. Recently a direct method for computing static electric and magnetic fields was reported [11] that uses the outer boundary compatibility relation as an explicit boundary condition.

\section{Compatibility relations and edge elements}

An example illustrating the need to include normal flux continuity conditions in a finite-element method using edge elements is given in [12]. The example shows that not including the normal flux continuity in the formulation allows the solution to contain "spurious solutions".

\section{E. Compatibility relations and face elements}

No examples illustrating the need to include tangential continuity conditions in a finite-element formulation 
when face expansions are used are presently available in the literature. It is, however, easy to construct such an example using the one for edge elements and normal flux continuity given in [12], and interchanging the roles of tangential and normal continuity.

\section{The Computational Costs}

As regards the computational costs of imposing the compatibility relations, we mention that they usually cause a significant increase in the connectivity of the system matrices. An additional difficulty is that some of the conditions even cause the matrices to lose their symmetry. Because of this, the storage requirements increase as well as the number of FLOPS (floating point operations) per CG (conjugent gradient) iteration step. A computational advantage of the inclusion of the compatibility conditions is that they improve the condition of the system matrix significantly, resulting in a much lower number of iterations being required before reaching convergence. Taking these effects together, the inclusion of the compatibility relations usually produces a more efficient code that yields more accurate results (for the same mesh).

\section{CONCLUSIONS}

When the electromagnetic field equations are solved numerically using expansions that do not themselves exactly satisfy these equations, which is the case in the finite-element method, it is necessary to include the compatibility relations in the formulation in order to obtain correct results. Attempts to solve this difficulty by using edge elements merely complicate the situation by introducing the need to impose additional compatibility relations. The importance of including the compatibility conditions is illustrated by reviewing a number of examples that are found in the literature. These examples demonstrate that erroneous (spurious) solutions due to not modeling certain compatibility conditions may, or may not, show up, depending on the type of problem, i.e. either on the configuration or on the time dependencies at hand.

In summary, we conclude that we have presented the electromagnetic field compatibility relations. To obtain reliable computational results from finite-element methods for solving the electromagnetic field equations, these relations should be made a part of the formulation of the problem.

\section{ACKNOWLEDGMENT}

The research reported in this paper has been financially supported through Research Grants from the Stichting Fund for Science, Technology and Research (a companion organization to the Schlumberger Foundation in the U.S.A.), from Schlumberger-Doll Research, Ridgefield, CT, U.S.A., from Etudes et Productions Schlumberger, Clamart, France, and from Schlumberger Cambridge Research Limited, Cambridge, England. This support is gratefully acknowledged.

\section{REFERENCES}

[1] A. E. H. Love, A Treatise on the Mathernatical Theory of Elasticity, The Syndics of the Cambridge University Press, London, 1959, p. 49.

[2] G. Mur, "The finite-element modeling of three-dimensional time harmonic electromagnetic fields in inhomogeneous media," Radio Science, vol. 26, No. 1, pp. 275-280, JanuaryFebruary 1991.

[3] G. Mur, "The finite-element modeling of three-dimensional time-domain electromagnetic fields in strongly inhomogeneous media," IEEE Trans. on Magnetics, vol. MAG-28, No. 2, pp. 1130-1133, March 1992.

[4] J. P. Webb, "Edge elements and what they can do for you," IEEE Trans. on Magnetics, vol. MAG-29, no. 2, pp. 14601465, March 1993.

[5] J. A. Stratton, Electromagnetic Theory, McGraw-Hill Book Company, New York, 1941, p. 486.

[6] I. D. Mayergoyz and J. D'Angelo, "A new point of view on the mathematical structure of Maxwell's equations," IEEE Trans. on Magnetics, vol. MAG-29, No. 2, pp. 1315-1320, March 1993.

[7] G. Mur, "The finite-element modeling of three-dimensional time-harmonic electromagnetic fields in anisotropic and strongly inhomogeneous media," COMPEL, vol. 9, 1990, Supplement A, pp. 83-86.

[8] G. Mur, "The finite-element modelling of three-dimensional electromagnetic fields using edge and nodal elements," IEEE Trans. on Antennas and Propagation, vol. 41, No. 7, pp. 948953, July 1993.

[9] X. Yuan, D. R. Lynch and K. Paulsen, "Importance of normal field continuity in inhomogeneous scattering calculations," JEEE Trans. on Microwave Theory and Techniques, vol. 39, No. 4, pp. 638-642, April 1991.

[10] G. Mur, "The FEMAX Finite-Element Package for Computing Three-Dimensional Time-Domain Electromagnetic Fields in Inhomogeneous Media," in: Software Applications in Electrical Engineering, Ed. P. P. Silvester, Computational Mechanics Publications, Southampton, 1993, pp. 187-194

[11] S. Sachdev and Z. J. Cendes, "Combined tangential-normal vector elements for computing electric and magnetic fields," IEEE Trans. on Magnetics, vol. MAG-29, No. 2, pp. 14561459, March 1993.

[12] G. Mur, "Edge elements, their advantages and their disadvantages," IEEE Trans. on Magnetics, this issue. 